\title{
Superradiance without inversion in a cavity: Effects of cavity losses and relaxation in the system of emitters
}

\author{
Igor Ryzhov ${ }^{1, *}$, Nikolai Vasil'ev ${ }^{1}$, and Victor Malyshev ${ }^{2}$ \\ ${ }^{1}$ A.I. Hertsen State Pedagogical University of Russia, St.-Petersburg, 191186 Russia \\ ${ }^{2}$ Zernike Institute for Advanced Materials, University of Groningen, 9747 AG Groningen, \\ The Netherlands
}

\begin{abstract}
We investigate theoretically inversionless superradiance of an ensemble of three-level emitters (with $\Lambda$-scheme of operating transition), placed in a cyclic cavity. Effects of cavity losses and emitter's relaxation on the dynamics of superradiance are studied.
\end{abstract}

The standard Dicke superradiance (SR) requires an initial population inversion of the operating transition to occur [1]. In the case of multilevel emitters (in particular, three-level atoms with the $\Lambda$-scheme of operating transitions considered here), this limitation is not crucial anymore: SR is possible even if the initial population of the upper level is smaller than the population of the doublet (SR without inversion, SRWI) [2-4]. In Ref. [2], it has been found that SRWI of an assembly of three-level $\Lambda$-emitters in a high- $Q$ cavity shows a rich optical dynamics (from regular to chaotic), depending on the population of the upper level $\alpha$ and splitting of the doublet $\omega_{21}$. These results have been obtained for a Hamiltonian system, i.e. neglecting the cavity losses and relaxation of coherence in the system of emitters. In reality, however, these factors are always present.

In this paper, we investigate theoretically the influence of relaxation on the regimes of SRWI found in Ref. [2] (see Ref. [4b] for details). In the Hamiltonian case and at a small doublet splitting, the SRWI dynamics represents a comb of chaotically repeating pulses with a repetition time on the order of the period of oscillations of the low-frequency coherence, $2 \pi / \omega_{21}$ (see Fig. 1a). Just this regime of SRWI is the goal of our study.

1. Cavity losses. This channel of SR relaxation is characterized by the SR lifetime in the cavity $T_{c}$. Figure $1 \mathrm{~b}$ shows an example of the SRWI dynamics for $T_{c}=50 \Omega^{-1}$, where $\Omega$ is the cooperative frequency introduced by Arecchi and Courtens [4b]; $\Omega$ determines the characteristic scale for the SR field and time. As is seen, the effect of cavity losses consists of, first, eliminating that part of the Hamiltonian SR pulse (shown in Fig.1a) which resides at times $t>T_{c}$, and second, decreasing the intensity of the remaining part. It should be noticed that the latter retains patterns of the Hamiltonian pulse.

Corresponding author: igoryzhov@yandex.ru 


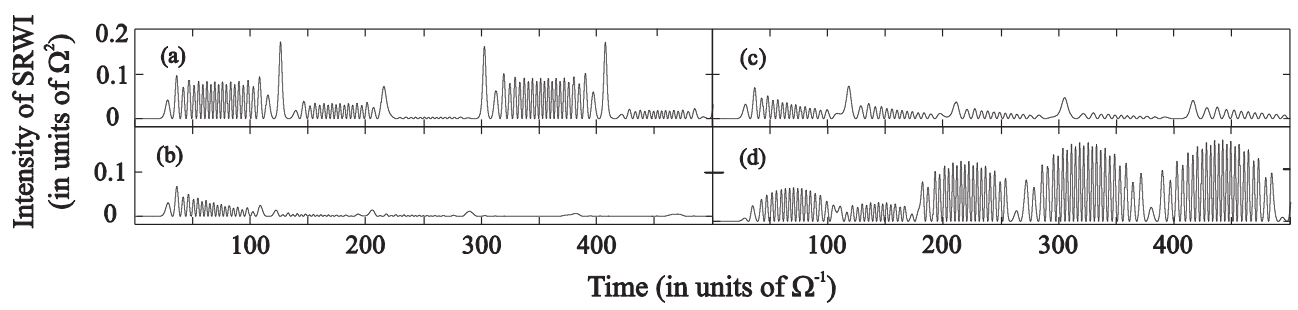

Fig. 1. (a) - The Hamiltonian SRWI pulse; (b) $-T_{c}=50 \Omega^{-1}, T_{2}=T_{2}{ }^{*}=\infty$; (c) $-T_{2}=50 \Omega^{-1}, T_{2}=T_{2}{ }^{*}=$ $\infty$; (d) $-T_{2}{ }^{*}=1000 \Omega^{-1} . T_{c}=T_{2}=\infty$. The initial population of the upper level $\alpha=0.3$, расщепление дублета $\omega_{21}=0.05 \Omega$.

2. Homogeneous broadening. This type of broadening is characterized by the time $T_{2}$ and results in the relaxation of coherence of an individual emitter. In an open system, this leads to decreasing the SR signal along with shortening $T_{2}$. In the case of a cavity-assisted SRWI, the global effect of $T_{2}$ also consists of inhibition of SRWI for time moments $t>T_{2}$. However, it appears that even for $t>T_{2}$, there exists a visible signal (see Fig. 1b). We associate this effect with two facts: (i) the SR field although decreases due to the emitter's dephasing, but does not escape the cavity because of a high quality of the latter, (ii) the low frequency coherence does not decay in our model and provides an additional source for the highfrequency coherence. In combination, these two factors result in a partial revival of the SRWI signal for $t>T_{2}$.

3. Inhomogeneous broadening. This type of broadening differs from its homogeneous counterpart: the phase of each emitter is preserved and only the collective response decays with time. The phases of the emitters can in principle be inverted by applying a coherent pulse. If, however, the $Q$ factor of the cavity is high enough. i.e. the SR field retains in the cavity for a long time, quasi-periodically/chaotically repeating combs of the SR pulses (like in our case) play the role of coherent trains, inverting the emitters' phases. Figure 1d shows the results of calculations of the SRWI dynamics for different values of the inhomogeneous relaxation time $T_{2}{ }^{*}$. As is seen, even a significantly large (on the computation scale) time $T_{2}{ }^{*}$ leads to a noticeable change in the shape of the SRWI pulse as compared with an ideal (Hamiltonian) system (Fig. 1a). We attribute such a strong effect of $T_{2}{ }^{*}$ on the SR pulse to the fact that the system resides at the state of dynamic chaos already in the ideal (Hamiltonian) case. Therefore, even small variations in the emitters' phases during emission lead to an essentially different scenario of the system's optical dynamics, preserving, however, its chaotic nature. For shorter $T_{2}{ }^{*}$, the dynamics of SRWI experiences even stronger changes.

Crystals doped with rare-earth ions (e.g., $\mathrm{LaF}_{3}: \mathrm{Pt}^{3+}, \mathrm{Y}_{2} \mathrm{SiO}_{5}: \mathrm{Pr}^{3+}, \mathrm{Y}_{2} \mathrm{SiO}_{5}: \mathrm{Eu}^{3+}$, and others) are those real objects in which the conditions for the observation of the nonstandard regimes of the cavity-assisted SRWI can be realized [4b].

This work was supported by the Russian Foundation for Basic Research (grant \#15-02-08369).

\section{References}

[1] R.H. Dicke, Phys. Rev. 93, 99 (1954)

[2] A.I. Zaitsev, V.A. Malyshev, E.D. Trifonov, and I.V. Ryzhov, JETP 88, 278 (1999)

[3] I.V. Ryzhov, A.I. Zaitsev, and E.V. Shuval-Sergeeva, Opt. Spectrosc. 112, 604 (2012)

[4] I.V. Ryzhov, N.A. Vasil'ev, I.S. Kosova, M.D. Shtager, and V.A. Malyshev, (a) Opt. Spectrosc. 120, 440 (2016); (b) JETP 124, 683 (2017) 